\title{
ON CUBIC TORSORS, BIEXTENSIONS AND SEVERI-BRAUER VARIETIES OVER ABELIAN VARIETIES
}

\author{
NATHAN GRIEVE
}

\begin{abstract}
We study homogeneous irreducible Severi-Brauer varieties over an Abelian variety $A$. Such objects were classified by Brion [5]. Here we interpret that result within the context of cubic structures and biextensions for certain $\mathbb{G}_{m}$-torsors over finite subgroups of A. Our results build on the theory of Breen, [3], and Moret-Bailly [15].
\end{abstract}

\section{INTRODUCTION}

Let $A$ be an Abelian variety, over an algebraically closed field $\mathbf{k}$, and let

$$
\operatorname{Br}(A):=\mathrm{H}_{\mathrm{et}}^{2}\left(A, \mathbb{G}_{m}\right)
$$

be its cohomological Brauer group. Fix a positive integer $r$ with $p:=\operatorname{char}(\mathbf{k}) \nmid r$. Our purpose here is to study $\operatorname{Br}(A)[r]$, the $r$-torsion subgroup of $\operatorname{Br}(A)$. In doing so, we apply work of Brion, [5], which builds on previous work of Atiyah, [1], Mumford [20], Berkovich [2], Mukai, [16], and others.

As in [5], we say that a Severi-Brauer variety over $A$ is homogeneous if it is invariant under pullback by all translations of elements of $A$. A homogeneous Severi-Brauer variety is irreducible if it contains no nontrivial proper homogeneous subbundle. In Section 6, we give a complete proof of the following result which was observed by Brion. It applies earlier work of Berkovich [2].

Theorem 1.1 ([5, page 2497]). Each class in $\operatorname{Br}(A)[r]$ may be represented by a homogeneous irreducible Severi-Brauer variety.

Second, we study cubic structures on $\mathbb{G}_{m}$-torsors over subgroups $\mathrm{K} \subseteq A[n]$, of the group of $n$-torsion points of $A$. We require that $\# \mathrm{~K}=n^{2}$ and $p \nmid n$. Our result identifies a class of such cubic torsors which correspond to the homogeneous irreducible $\mathbb{P}^{n-1}$-bundles over A. We say that such cubic torsors are nondegenerate and refer to Definition 8.4 for precise details. Using this terminology, our main novel conceptual result is

Theorem 1.2. Each class in $\operatorname{Br}(A)[r]$ may be represented by a nondegenerate cubic torsor.

Mathematics Subject Classification (2020): 14K05, 14F22.

This article has been published in São Paulo Journal of Mathematical Sciences. The final published version is available online at: https://doi.org/10.1007/s40863-021-00250-3. 
One step in our proof of Theorem 1.2 involves a reformulation of the basic structure theorems, for homogeneous projective bundles over $A$, which were obtained by Brion in [5]. More precisely, a inite type group scheme is anti-affine if it admits no global non-constant regular functions. In particular, such a group is smooth, connected and commutative. For our purposes, an anti-affine extension of an Abelian variety $A$, is a short exact sequence of commutative group schemes

$$
1 \rightarrow H \rightarrow G \stackrel{\pi}{\rightarrow} A \rightarrow 1
$$

in which the group $G$ is anti-affine and $H$ is affine.

In [5], Brion characterized the homogeneous projective bundles over $A$, in terms of antiaffine extensions (1.1) together with a faithful projective representation of the commutative group scheme $H$.

Theorem 1.3 ([5, Theorem 2.1]). Let $\mathbf{k}$ be an algebraically closed field. Let $\mathrm{P}$ be a $\mathbb{P}^{n-1}$ bundle over an Abelian variety $A$ and assume that $n$ is not divisible by the characteristic of k. The following assertions hold true.

(i) The $\mathbb{P}^{n-1}$-bundle $\mathrm{P}$ is homogeneous if and only if there exists an anti-affine extension (1.1) together with a faithful homomorphism

$$
\rho: H \rightarrow \mathrm{PGL}_{n}
$$

such that $\mathrm{P}$ is the associated bundle

$$
\mathrm{P} \simeq G \times{ }^{H} \mathbb{P}^{n-1} \rightarrow G / H=A .
$$

Here, in (1.3), $H$ acts on $\mathbb{P}^{n-1}$ via the representation (1.2). The anti-affine extension (1.1) is unique and the projective representation (1.2) is unique up to conjugacy in $\mathrm{PGL}_{n}$. Further, the $\mathrm{PGL}_{n}$-torsor that corresponds to the bundle (1.3) is the $\mathrm{PGL}_{n^{-}}$ bundle

$$
G \times{ }^{H} \mathrm{PGL}_{n} \rightarrow A
$$

the corresponding Azumaya algebra is

$$
\mathcal{A}=\left(\pi_{*}\left(\mathcal{O}_{G}\right) \otimes M_{n}\left(\mathcal{O}_{A}\right)\right)^{H} .
$$

Finally, the A-automorphism group of such a homogeneous bundle (1.3) is isomorphic to the centralizer of $H$ in the projective linear group $\mathrm{PGL}_{n}$.

(ii) If $\mathrm{P}$ is homogeneous, then its homogeneous projective subbundles are exactly the bundles

$$
G \times{ }^{H} S \rightarrow A
$$

where $S \subseteq \mathbb{P}^{n-1}$ is an $H$-stable linear subspace. Further, if $\left(\mathrm{P}_{1}, \mathrm{P}_{2}\right)$ is a pair of disjoint $\mathbb{P}^{n_{i}-1}-$ subbundles of $\mathrm{P}$, with $n_{1}+n_{2}=n$, then both $\mathrm{P}_{1}$ and $\mathrm{P}_{2}$ are homogeneous subbundles of $\mathrm{P}$. 
In the present article, we relate the conceptual framework of [3] and [15] to the classification that is given in [5]. In doing so, we obtain a description of $\operatorname{Br}(A)[r]$ that is in terms of cubic structures and biextensions. The fact that the Theorem of the Cube is related to the Brauer group is well-known [11]. We refer to the works [23] and [6], for example, as a starting point to the study of Brauer groups of Abelian varieties over non-algebraically closed fields. We also mention the work [22] which provides additional motivation for further research in the direction that we pursue here.

Our main result is Theorem 9.1. It interprets the homogeneous irreducible $\mathbb{P}^{n-1}$-bundles over $A$, for $p \nmid n$, in terms of the works [3] and [15]. We also use Theorem 9.1, in conjunction with Theorem 1.1, to prove Theorem 1.2, Both of these results, Theorem 9.1 and Theorem 1.2, together with Theorem 1.1, which is due to Brion, have not, at present, been adequately discussed in the literature.

Notations and other conventions. We fix an algebraically closed base field $\mathbf{k}$ and let $p:=\operatorname{char}(\mathbf{k})$. Unless explicitly stated otherwise, we fix positive integers $n$ and $r$ and assume that they are not divisible by $p$. When no confusion is likely, we fail to distinguish between the concepts of $\mathbb{G}_{m}$-torsors, total spaces of line bundles and invertible sheaves. We consider Abelian varieties $A$ over $\mathbf{k}$. The dual of $A$ is denoted by $A^{t}$. If $x \in A$, then $\tau_{x}: A \rightarrow A$ denotes translation by $x$ in the group law. If $m \in \mathbb{Z}$, then $A[m]$ denotes the kernel of the morphism $[m]_{A}: A \rightarrow A, x \mapsto m x$. This is the set of $m$-torsion points of $A$. By a $\mathbb{P}^{n-1}{ }_{-}$ bundle over $A$, or equivalently a rank $n$ Severi-Brauer variety over $A$, we mean a proper flat morphism $\mathrm{P} \rightarrow A$ with fibers at all closed points, $x \in A$, projective space $\mathbb{P}^{n-1}$. Finally, if $H$ is a finite commutative $\mathbf{k}$-group scheme, then $H^{t}$ is its Cartier dual.

Acknowledgements. Portions of this work were completed during my time as a postdoctoral fellow at Michigan State University. It benefited from visits to ICERM (Providence), during June 2019, and BIRS (Banff), during November 2019. I thank Igor Rapinchuk and colleagues for their interest and discussions on topics related to this work. Further, I thank the Natural Sciences and Engineering Research Council of Canada for supporting my work through my grants DGECR-2021-00218 and RGPIN-2021-03821. Finally, I thank anonymous referees for their careful reading, corrections, helpful comments and suggestions.

\section{Preliminaries about anti-AfFine extensions}

In general, the structure of each such anti-affine extension (1.1) depends on the characteristic of the base field. Indeed, the following theorem, from [4], gives a description of the set of isomorphism classes of anti-affine groups over a given Abelian variety. In what follows, by an anti-affine group over an Abelian variety $A$, is meant an exact sequence of commutative group schemes

$$
1 \rightarrow H \rightarrow G \rightarrow A \rightarrow 1
$$

as in (1.1), with $G$ an anti-affine group and $H$ an affine group. 
Theorem 2.1 ([4, Theorem 2.7]). Let $A$ be an Abelian variety over an algebraically closed field $\mathbf{k}$ with $p:=$ char $(\mathbf{k})$. Then, the following assertions hold true.

(i) If $p>0$, then the set of isomorphism classes of anti-affine groups over $A$ is in bijective correspondence with the set of sublattices of $A^{t}(\mathbf{k})$.

(ii) If $p=0$, then the set of isomorphism classes of anti-affine groups over $A$ are in bijective correspondence with the set of pairs $(\Lambda, V)$, where $\Lambda$ is a sublattice of $A^{t}(\mathbf{k})$ and where $V$ is a subvector space of $\mathrm{H}^{1}\left(A, \mathcal{O}_{A}\right)$.

While the overall structure of such anti-affine extensions (1.1) depends on $p$, what remains true independently of the characteristic, is that they are classified by specifying a finite subgroup scheme $\mathrm{K} \subseteq A$ together with an anti-affine group over the Abelian variety $\left(A^{t} / \mathrm{K}^{t}\right)^{t}$. For later use, we make explicit mention of this fact. We include a proof for completeness.

Proposition 2.2. The anti-affine extensions (1.1) are classified by specifying a finite subgroup scheme $\mathrm{K} \subseteq A$ together with an anti-affine group over the Abelian variety $\left(A^{t} / \mathrm{K}^{t}\right)^{t}$. Further, given such an anti-affine extension (1.1) if $H$ is finite, then the anti-affine group $G$ is an Abelian variety.

Proof. First, suppose given a finite subgroup scheme $\mathrm{K} \subseteq A$ together with an anti-affine group $G$ over the Abelian variety $\left(A^{t} / \mathrm{K}^{t}\right)^{t}$. Let $H$ be the kernel of the induced map

$$
G \rightarrow\left(A^{t} / \mathrm{K}^{t}\right)^{t} \rightarrow A^{t t}=A
$$

Then we obtain an anti-affine extension of the form (1.1).

For the converse, if $G$ is an anti-affine group over $A$ and fitting into an extension as in (1.1), then the morphism $\pi$ factors through

$$
B:=\operatorname{Alb}(G)=G / G_{\mathrm{aff}},
$$

the Albanese variety of $G$. Let $\mathrm{K}$ be the Cartier dual of $\mathrm{K}^{t}$, the kernel of the induced isogney

$$
A^{t} \rightarrow B^{t}
$$

Then $\mathrm{K}$ is the finite subgroup of $A$ that is determined by the anti-affine extension (1.1). By double duality, arguing as in (7.3), (7.4), (7.5), $G$ is an anti-affine group over $\left(A^{t} / \mathrm{K}^{t}\right)^{t}$.

For the remaining assertion, if $H$ is finite, then $G_{\text {aff }}$, the largest smooth connected affine normal subgroup of $G$, is trivial. It follows that $G$ is isomorphic to its Albanese variety. Hence, $G$ is an Abelian variety.

Together, Theorem 1.3 and Proposition 2.2 allow for the following characterization of homogenous bundles over $A$.

Corollary 2.3. Let $\mathrm{P}$ be a rank $n$ Severi-Brauer variety over an Abelian variety $A$ and assume that $n$ is not divisible by the characteristic of $\mathbf{k}$. Then $\mathrm{P}$ is homogeneous if and only 
if there exists a finite subgroup $\mathrm{K} \subseteq A$ and an anti-affine group $G$ over the Abelian variety $\left(A^{t} / \mathrm{K}^{t}\right)^{t}$ such that if $H$ is the kernel of the induced map $G \rightarrow A$, then

$$
\mathrm{P} \simeq G \times{ }^{H} \mathbb{P}^{n-1} \rightarrow A
$$

for some faithful projective representation $\rho: H \rightarrow \mathrm{PGL}_{n}$.

Proof. By Proposition 2.2, the anti-affine extensions (1.1) are classified by finite subgroups $\mathrm{K} \subseteq A$ together with anti-affine groups $G$ over $\left(A^{t} / \mathrm{K}^{t}\right)^{t}$. Thus, the conclusion that is desired by Corollary 2.3 follows upon combining Proposition 2.2 and Theorem 1.3 (i).

A homogeneous projective bundle is irreducible if it contains no nontrival proper homogeneous subbundle. The classification of homogeneous irreducible projective bundles involves the weight 1 representations of nondegenerate theta groups, [17], [15. For example, Theorem 1.3. Proposition 2.2 and Corollary 2.3 , together with the theory of nondegenerate theta groups, allow for the homogeneous irreducible projective bundles to be described as in Corollary 2.4 below. Again, we include a short proof for completeness. We discuss the theory of non-degenerate theta groups in Sections 5 and 9.

Corollary 2.4 ([5, Proposition 3.1]). The homogeneous irreducible rank $n$ Severi-Brauer varieties over $A$, for $p \nmid n$, are classified by pairs $(H, e)$, where

$$
H \subseteq A[n]
$$

is a subgroup of rank $n^{2}$ and where

$$
e: H \times H \rightarrow \mathbb{G}_{m}
$$

is a nondegenerate alternating pairing.

Proof. By the theory of nondegenerate theta groups, [17, page 293], see also Sections 5 and 9, the nondegenerate alternating map (2.2) specifies an irreducible weight one representation of a nondegenerate theta group over $H$. It also induces an isomorphism

$$
H \simeq H^{t} .
$$

The desired anti-affine group over $A$ is the Abelian variety $\left(A / H^{t}\right)^{t}$.

\section{Equivalent descriptions of Azumaya algebras and Severi-Brauer VARIETIES}

Let $X$ be a nonsingular projective variety over an algebraically closed field $\mathbf{k}$ and let $p:=\operatorname{char}(\mathbf{k})$. There are many equivalent descriptions of Azumaya algebras over $X$. To begin with, by an Azumaya algebra over $X$, we mean an $\mathcal{O}_{X}$-algebra $\mathcal{A}$ which is a coherent $\mathcal{O}_{X}$-module and which has the property that its stalk $\mathcal{A}_{x}$ at all closed points $x \in X$ is an Azumaya algebra over the local ring $\mathcal{O}_{X, x}$. In particular, $\mathcal{A}_{x}$ is Azumaya over $\mathcal{O}_{X, x}$ for all points $x \in X$. Especially, $\mathcal{A}$ is a locally free $\mathcal{O}_{X}$-module of finite square rank. Equivalently 
(i) the algebra $\mathcal{A}$ is a locally free $\mathcal{O}_{X}$-module and

$$
\left.\mathcal{A}\right|_{x}:=\mathcal{A}_{x} \otimes \kappa(x)
$$

is a central simple $\kappa(x)$-algebra for each $x \in X$;

(ii) the algebra $\mathcal{A}$ is a locally free $\mathcal{O}_{X}$-module and the canonical homomorphism

$$
\mathcal{A} \otimes_{\mathcal{O}_{X}} \mathcal{A}^{\circ} \rightarrow \mathcal{E}_{n d_{\mathcal{O}_{X}}}(\mathcal{A})
$$

is an isomorphism; and

(iii) there exists a covering $\left\{U_{i} \rightarrow X\right\}_{i \in I}$, for the étale topology on $X$, so that for each $i$, there exists $r_{i}$ with the property that

$$
\mathcal{A} \otimes_{\mathcal{O}_{X}} \mathcal{O}_{U_{i}} \simeq M_{r_{i}}\left(\mathcal{O}_{U_{i}}\right)
$$

see for example [13, Chapter IV].

The geometric study of Azumaya algebras is achieved via their bijective correspondence with $\mathrm{PGL}_{n}$-torsors and $\mathbb{P}^{n-1}$-bundles over $X$. For instance, under this bijective correspondence, each such rank $n^{2}$ Azumaya algebra $\mathcal{A}$ has the form

$$
\mathcal{A} \simeq\left(\pi_{*}\left(\mathcal{O}_{Y}\right) \otimes M_{n}\left(\mathcal{O}_{X}\right)\right)^{\mathrm{PGL}_{n}}
$$

for

$$
\pi: Y \rightarrow X
$$

the corresponding $\mathrm{PGL}_{n}$-torsor; the corresponding $\mathbb{P}^{n-1}$-bundle is then

$$
\mathrm{P} \simeq Y \times{ }^{\mathrm{PGL}_{n}} \mathbb{P}^{n-1} \rightarrow X .
$$

In particular, the structure morphism (3.3) is a proper flat morphism with $\mathbb{P}^{n-1}$-fibres over closed points of $X$.

Example 3.1 ([5, page 2477]). Recall the product of two $\mathbb{P}^{n_{i}-1}$-bundles

$$
P_{i} \rightarrow X
$$

for $i=1,2$. Let

$$
Y_{i} \rightarrow X
$$

be the $\mathrm{PGL}_{n_{i}}$-bundles corresponding to $\mathrm{P}_{i}$. Then

$$
\mathrm{P}_{1} \cdot \mathrm{P}_{2} \rightarrow X
$$

is the $\mathbb{P}^{n_{1} n_{2}-1}$-bundle that is obtained from the $\mathrm{PGL}_{n_{1}} \times \mathrm{PGL}_{n_{2}}$-torsor

$$
Y_{1} \times_{X} Y_{2} \rightarrow X
$$

via the tensor product extension of structure groups

$$
\mathrm{PGL}_{n_{1}} \times \mathrm{PGL}_{n_{2}} \rightarrow \mathrm{PGL}_{n_{1} n_{2}} .
$$

Next, we consider Example 3.1 for the particular case of homogeneous projective bundles over a given Abelian variety. 
Example 3.2 ([5, Remark 2.2]). If

$$
\mathrm{P}_{i}=G_{i} \times^{H_{i}} \mathbb{P}^{n_{i}-1}
$$

for $i=1,2$, are homogeneous $\mathbb{P}^{n_{i}-1}$-bundles over and Abelian variety $A, p \nmid n_{i}$, corresponding to anti-affine extensions

$$
1 \rightarrow H_{i} \rightarrow G_{i} \rightarrow A \rightarrow 1
$$

and faithful projective representations

$$
\rho_{i}: H_{i} \rightarrow \mathrm{PGL}_{n_{i}},
$$

then the product bundle $\mathrm{P}_{1} \cdot \mathrm{P}_{2}$ is homogeneous. It has the form

$$
\mathrm{P}_{1} \cdot \mathrm{P}_{2}=G \times{ }^{H} \mathbb{P}^{n_{1} \cdot n_{2}-1}
$$

for

$$
G \subseteq G_{1} \times_{A} G_{2}
$$

the largest anti-affine subgroup,

$$
H:=\left(H_{1} \times H_{2}\right) \bigcap G
$$

and projective representation

$$
\rho=\left.\left(\rho_{1} \otimes \rho_{2}\right)\right|_{H}
$$

To conclude this section, we recall the cohomological description of Severi-Brauer varieties. Let $n$ be a positive integer that is not divisible by $p$. Recall that the pointed cohomology set $\mathrm{H}_{\mathrm{et}}^{1}\left(X, \mathrm{PGL}_{n}\right)$ classifies isomorphism classes of rank $n^{2}$ Azumaya algebras over $X$. Equivalently, $\mathrm{H}_{\text {et }}^{1}\left(X, \mathrm{PGL}_{n}\right)$ classifies $\mathrm{PGL}_{n}$-torsors over $X$. The class of such an Azumaya algebra in

$$
\operatorname{Br}(X)=\mathrm{H}_{\mathrm{et}}^{2}\left(X, \mathbb{G}_{m}\right)
$$

is obtained via the boundary morphism

$$
\partial: \mathrm{H}_{\mathrm{et}}^{1}\left(X, \mathrm{PGL}_{n}\right) \rightarrow \operatorname{Br}(X)
$$

which is induced by the exact sequence

$$
1 \rightarrow \mathbb{G}_{m} \rightarrow \mathrm{GL}_{n} \rightarrow \mathrm{PGL}_{n} \rightarrow 1
$$

of étale sheaves of groups.

\section{Cyclic Azumaya algebras and the Brauer group}

In this section, we recall the most basic properties of $\operatorname{Br}(X)[r]$, the $r$-torsion part of the Brauer group of a nonsingular projective variety $X$. Here $r$ is a positive integer which is not divisible by the characteristic of the algebraically closed base field $\mathbf{k}$. The main point is Theorem 4.4, which, among other things, says that the group $\operatorname{Br}(X)[r]$ is generated by the Hilbert symbols $\{\alpha, \beta\}_{r}$. Equivalently, $\operatorname{Br}(X)[r]$ is generated by the classes of $\mathcal{A}(\alpha, \beta)$, the cyclic algebras determined by $r$-torsion line bundles $\alpha$ and $\beta$ on $X$. What we describe here 
is based on [2], [13] and [12]. Corollary 4.5] and Example 4.6 illustrate the more specific case of Abelian varieties.

By a rigidified $r$-torsion line bundle on $X$, is meant an $r$-torsion line bundle $\alpha$ together with a fixed choice of isomorphism

$$
\mathcal{O}_{X} \stackrel{\sim}{\rightarrow} \alpha^{\otimes r}
$$

Let $\mu_{r}$ denote the multiplicative group of $r$ th roots of unity and fix a primitive $r$ th root of unity $\zeta \in \mu_{r}$. The collection of isomorphism classes of rigidified $r$-torsion line bundles may be identified with the étale cohomology group $\mathrm{H}_{\mathrm{et}}^{1}\left(X, \mu_{r}\right)$.

Fix a pair of rigidified $r$-torsion line bundles $(\alpha, \beta)$ on $X$. The cyclic algebra determined by $(\alpha, \beta)$ is then

$$
\mathcal{A}(\alpha, \beta):=\bigoplus_{0 \leq i, j \leq r-1} \alpha^{\otimes i} \otimes \beta^{\otimes j}
$$

In (4.1), the algebra structure depends on $\zeta$ and is determined by

$$
\left(\alpha^{\otimes i} \otimes \beta^{\otimes j}\right) \otimes\left(\alpha^{\otimes k} \otimes \beta^{\otimes \ell}\right) \stackrel{\zeta^{j k}}{\longrightarrow}\left(\alpha^{\otimes i} \otimes \alpha^{\otimes k}\right) \otimes\left(\beta^{\otimes j} \otimes \beta^{\otimes \ell}\right) \rightarrow \alpha^{\otimes q} \otimes \beta^{\otimes s},
$$

where

$$
i+k \equiv q \bmod r \text { and } j+\ell \equiv s \bmod r
$$

for $0 \leq q, s \leq r-1$.

The affine local structure of $\mathcal{A}(\alpha, \beta)$ is described by fixing affine open subsets $U \subseteq X$ which trivialize $\alpha$ and $\beta$. In particular

$$
\left.\alpha\right|_{U} \stackrel{\sim}{\rightarrow} \mathcal{O}_{U} e_{\alpha} \stackrel{\sim}{\rightarrow} \mathcal{O}_{U}=\left.\mathcal{O}_{X}\right|_{U}
$$

and

$$
\left.\beta\right|_{U} \stackrel{\sim}{\rightarrow} \mathcal{O}_{U} e_{\beta} \stackrel{\sim}{\rightarrow} \mathcal{O}_{U}=\left.\mathcal{O}_{X}\right|_{U}
$$

Here $e_{\alpha}$ and $e_{\beta}$ are local generators for $\left.\alpha\right|_{U}$ and $\left.\beta\right|_{U}$ respectively. Let

$$
\phi: \mathcal{O}_{X} \stackrel{\sim}{\rightarrow} \alpha^{\otimes r}
$$

and

$$
\psi: \mathcal{O}_{X} \stackrel{\sim}{\rightarrow} \beta^{\otimes r}
$$

denote the fixed rigidifications of $\alpha^{\otimes r}$ and $\beta^{\otimes r}$. There exists units $a, b \in \Gamma\left(U, \mathcal{O}_{U}\right)$ with the properties that

$$
\left.a \cdot \phi(1)\right|_{U}=e_{\alpha}^{\otimes r}
$$

and

$$
\left.b \cdot \psi(1)\right|_{U}=e_{\beta}^{\otimes r} .
$$

It follows that $\left.\mathcal{A}(\alpha, \beta)\right|_{U}$ is isomorphic, as an $\mathcal{O}_{U}$-algebra, to the $\mathcal{O}_{U}$-algebra which is generated by the elements $\mathbf{e}_{\alpha}$ and $\mathbf{e}_{\beta}$ subject to the relations that

$$
\mathbf{e}_{\alpha}^{r}=a, \mathbf{e}_{\beta}^{r}=b \text { and } \mathbf{e}_{\alpha} \cdot \mathbf{e}_{\beta}=\zeta \mathbf{e}_{\beta} \cdot \mathbf{e}_{\alpha} .
$$

Note that $\mathcal{A}(\alpha, \beta)$ is an Azumaya algebra of rank $r^{2}$ over $A$. 
As in [12, we consider a concept of Hilbert symbol for a pair of $r$-torsion line bundles.

Definition $4.1([12])$. Let $\{\alpha, \beta\}_{r}$ denote the class of $\mathcal{A}(\alpha, \beta)$ in

$$
\operatorname{Br}(X)[r]=\mathrm{H}_{\text {et }}^{2}\left(X, \mathbb{G}_{m}\right)[r] .
$$

We say that $\{\alpha, \beta\}_{r}$ is the Hilbert symbol of $\alpha$ and $\beta$.

The vanishing of such Hilbert symbols $\{\alpha, \beta\}_{r}$ detects when the cyclic algebra $\mathcal{A}(\alpha, \beta)$ has trivial class in the Brauer group.

Proposition $4.2([12])$. Let $(\alpha, \beta)$ be a fixed pair of rigidified $r$-torsion line bundles on $X$. The following two assertions are equivalent.

(i) The cyclic algebra $\mathcal{A}(\alpha, \beta)$ has the form

$$
\mathcal{A}(\alpha, \beta) \simeq \mathcal{E} n d(\mathcal{E})
$$

for some vector bundle $\mathcal{E}$ on $X$.

(ii) The Hilbert symbol $\{\alpha, \beta\}_{r}$ determined by $(\alpha, \beta)$ is trivial

$$
\{\alpha, \beta\}_{r}=0 \text {. }
$$

Proof. This can be checked locally as noted in [12, Proposition 1.5].

By analogy with the Hilbert tame symbols that arise in the Milnor K-theory of fields, [8, Chapter 7], the Hilbert symbols, in the sense of Definition 4.1, exhibit the following properties.

Proposition 4.3 ([12]). Let $\alpha, \beta$ and $\gamma$ be a collection of rigidified $r$-torsion line bundles on $X$. The following properties hold true

(i) $\{\alpha \otimes \beta, \gamma\}_{r}=\{\alpha, \gamma\}_{r}+\{\beta, \gamma\}_{r}$;

(ii) $\{\alpha, \beta \otimes \gamma\}_{r}=\{\alpha, \beta\}_{r}+\{\alpha, \gamma\}_{r}$; and

(iii) $\{\alpha, \beta\}_{r}+\{\beta, \alpha\}_{r}=0$.

Proof. This can also be checked locally via a cup-product calculation, [12, Corollary 2.6].

The first main result for $r$-torsion in the Brauer group of $X$ is stated in the following way.

Theorem 4.4 ([13, Chapter IV]). Let $X$ be a nonsingular projective variety over an algebraically closed field $\mathbf{k}$ and fix a positive integer $r$ which is not divisible by the characteristic of $\mathbf{k}$. In this context, the r-torsion part of the Brauer group $\operatorname{Br}(X)$ is generated by the Hilbert symbols. Furthermore, the group $\operatorname{Br}(X)[r]$ sits in an exact sequence

$$
0 \rightarrow \operatorname{Pic}(X) / r \operatorname{Pic}(X) \rightarrow \mathrm{H}_{\mathrm{et}}^{2}\left(X, \mu_{r}\right) \rightarrow \operatorname{Br}(X)[r] \rightarrow 0 .
$$

Proof. The key point is that this exact sequence is induced by the Kummer sequence

$$
1 \rightarrow \mu_{r} \rightarrow \mathbb{G}_{m} \stackrel{\cdot r}{\rightarrow} \mathbb{G}_{m} \rightarrow 1 .
$$

We refer to [13, Chapter IV] for more details. 
For the case of Abelian varieties, Theorem 4.4 takes a more explicit form.

Corollary 4.5 ([2], [12]). Let $A$ be a g-dimensional Abelian variety over an algebraically closed field $\mathbf{k}$. Let $\rho$ be the Picard number of $A$. In this context, the r-torsion part of $\operatorname{Br}(A)[r]$, for $r$ not divisible by the characteristic of $\mathbf{k}$, is a free $\mathbb{Z} / r \mathbb{Z}$-module of rank $\left(\begin{array}{c}2 g \\ 2\end{array}\right)-\rho$.

Proof. Since

$$
\mathrm{H}_{\mathrm{et}}^{2}\left(A, \mu_{r}\right)=\operatorname{Hom}\left(\bigwedge^{2} A[r], \mu_{r}\right),
$$

the conclusion of Corollary 4.5 follows from Theorem 4.4,

Example 4.6. As an illustration of Corollary 4.5, it is helpful to consider the case of a complex Abelian variety $A$ that is the product of elliptic curves. By [24], see also [25], the discussion that follows also allows for a description of the Brauer group of $\operatorname{Kum}(A)$, the nonsingular Kummer variety that is determined by $A$.

First, let $\mathfrak{h}$ denote the upper half plane and consider the case that

$$
A \simeq E_{1} \times E_{2}
$$

for elliptic curves

$$
E_{i} \simeq \mathbb{C} / \Lambda_{i}
$$

where

$$
\Lambda_{i}=\mathbb{Z} \oplus \tau_{i} \mathbb{Z}
$$

and $\tau_{i} \in \mathfrak{h}, i=1,2$. Then

$$
\begin{aligned}
\operatorname{Br}(A) & \simeq \operatorname{Hom}_{\mathbb{Z}}(T(A), \mathbb{Q} / \mathbb{Z}) \\
& \simeq \operatorname{Hom}_{\mathbb{Z}}(T(A), \mathbb{Z}) \otimes \mathbb{Q} / \mathbb{Z} \\
& \simeq \mathrm{H}^{2}(A, \mathbb{Z}) / \mathrm{NS}(A) \otimes \mathbb{Q} / \mathbb{Z}
\end{aligned}
$$

where

$$
T(A) \simeq \mathrm{NS}(A)^{\perp}
$$

is the transcendental lattice.

In order to determine the Néron-Severi group

$$
\mathrm{NS}(A) \simeq \mathrm{H}^{1,1}(A, \mathbb{Z}),
$$

let $x_{1}, \ldots, x_{4}$ be real coordinates corresponding to the dual basis of

$$
\begin{aligned}
\mathrm{H}_{1}(X, \mathbb{Z}) & =\mathrm{H}_{0}\left(E_{2}, \mathbb{Z}\right) \otimes \mathrm{H}_{1}\left(E_{1}, \mathbb{Z}\right) \oplus \mathrm{H}_{1}\left(E_{2}, \mathbb{Z}\right) \otimes \mathrm{H}_{0}\left(E_{1}, \mathbb{Z}\right) \\
& =\Lambda_{1} \oplus \Lambda_{2} .
\end{aligned}
$$

Let

$$
\omega_{1}=d x_{1}+\tau_{1} d x_{3}
$$

and

$$
\omega_{2}=d x_{3}+\tau_{2} d x_{4}
$$


Then

$$
\bar{\omega}_{1}=d x_{1}+\bar{\tau}_{1} d x_{2}
$$

and

$$
\bar{\omega}_{2}=d x_{3}+\bar{\tau}_{2} d x_{4}
$$

the ordered bases

$$
d x_{1} \wedge d x_{2}, d x_{1} \wedge d x_{3}, d x_{1} \wedge d x_{4}, d x_{2} \wedge d x_{3}, d x_{2} \wedge d x_{4}, d x_{3} \wedge d x_{4}
$$

and

$$
\omega_{1} \wedge \omega_{2}, \omega_{1} \wedge \bar{\omega}_{1}, \omega_{1} \wedge \bar{\omega}_{2}, \omega_{2} \wedge \bar{\omega}_{1}, \omega_{2} \wedge \bar{\omega}_{2}, \bar{\omega}_{1} \wedge \bar{\omega}_{2}
$$

for $\mathrm{H}^{2}(A, \mathbb{C})$, are related by the transition matrix

$$
\left(\begin{array}{cccccc}
0 & \frac{\bar{\tau}_{1} \bar{\tau}_{2}}{\left(\bar{\tau}_{1}-\tau_{1}\right)\left(\bar{\tau}_{2}-\tau_{2}\right)} & \frac{-\bar{\tau}_{1}}{\left(\bar{\tau}_{1}-\tau_{1}\right)\left(\bar{\tau}_{2}-\tau_{2}\right)} & \frac{-\bar{\tau}_{2}}{\left(\bar{\tau}_{1}-\tau_{1}\right)\left(\bar{\tau}_{2}-\tau_{2}\right)} & \frac{1}{\left(\bar{\tau}_{1}-\tau_{1}\right)\left(\bar{\tau}_{2}-\tau_{2}\right)} & 0 \\
\frac{\bar{\tau}_{1}-\tau_{1}}{\left(\bar{\tau}_{1}-\tau_{1}\right)^{2}} & 0 & 0 & 0 & 0 & 0 \\
0 & \frac{-\bar{\tau}_{1} \tau_{2}}{\left(\bar{\tau}_{1}-\tau_{1}\right)\left(\bar{\tau}_{2}-\tau_{2}\right)} & \frac{\tau_{1}}{\left(\bar{\tau}_{1}-\tau_{1}\right)\left(\bar{\tau}_{2}-\tau_{2}\right)} & \frac{-\tau_{2}}{\left(\bar{\tau}_{1}-\tau_{1}\right)\left(\bar{\tau}_{2}-\tau_{2}\right)} & \frac{-1}{\left(\bar{\tau}_{1}-\tau_{1}\right)\left(\bar{\tau}_{2}-\tau_{2}\right)} & 0 \\
0 & \frac{\tau_{1} \tau_{2}}{\left(\bar{\tau}_{1}-\tau_{1}\right)\left(\bar{\tau}_{2}-\tau_{2}\right)} & \frac{-\tau_{1}}{\left(\bar{\tau}_{1}-\tau_{1}\right)\left(\bar{\tau}_{2}-\tau_{2}\right)} & \frac{1}{\left(\bar{\tau}_{1}-\tau_{1}\right)\left(\bar{\tau}_{2}-\tau_{2}\right)} & \frac{1}{\left(\bar{\tau}_{1}-\tau_{1}\right)\left(\bar{\tau}_{2}-\tau_{2}\right)} & 0 \\
0 & 0 & 0 & 0 & 0 & \frac{\bar{\tau}_{2}-\tau_{2}}{\left(\bar{\tau}_{2}-\tau_{2}\right)^{2}} \\
0 & \frac{\tau_{1} \tau_{2}}{\left(\bar{\tau}_{1}-\tau_{1}\right)\left(\bar{\tau}_{2}-\tau_{2}\right)} & \frac{-\tau_{1}}{\left(\bar{\tau}_{1}-\tau_{1}\right)\left(\bar{\tau}_{2}-\tau_{2}\right)} & \frac{-\tau_{2}}{\left(\bar{\tau}_{1}-\tau_{1}\right)\left(\bar{\tau}_{2}-\tau_{2}\right)} & \frac{1}{\left(\bar{\tau}_{1}-\tau_{1}\right)\left(\bar{\tau}_{2}-\tau_{2}\right)} & 0
\end{array}\right)
$$

Among other things, this matrix allows for calculation of the Picard number $\rho(A)$. For instance, the Néron-Severi group $\mathrm{H}^{1,1}(A, \mathbb{Z})$ consists exactly of those forms

$$
a_{12} d x_{1} \wedge d x_{2}+a_{13} d x_{1} \wedge d x_{3}+a_{14} d x_{1} \wedge d x_{4}+a_{23} d x_{2} \wedge d x_{3}+a_{24} d x_{2} \wedge d x_{4}+a_{34} d x_{3} \wedge d x_{4}
$$

where $a_{12}, a_{13}, a_{14}, a_{23}, a_{24}, a_{34} \in \mathbb{Z}$ and the collection of integers $a_{13}, a_{14}, a_{23}, a_{24}$ satisfies the equation

$$
b_{13} \tau_{1} \tau_{2}-b_{14} \tau_{1}-b_{23} \tau_{2}+b_{24}=0,
$$

for unknown integers $b_{13}, b_{14}, b_{23}$ and $b_{24}$. In more explicit terms, the following assertions hold true.

- If $\rho(A)=2$, then $\mathrm{H}^{1,1}(A, \mathbb{Z})$ is spanned by the forms

(i) $d x_{1} \wedge d x_{2}$; and

(ii) $d x_{3} \wedge d x_{4}$.

- If $\rho(A)=3$, then $\mathrm{H}^{1,1}(A, \mathbb{Z})$ is spanned by the forms

(i) $d x_{1} \wedge d x_{2}$;

(ii) $d x_{3} \wedge d x_{4}$; and

(iii) $a_{13} d x_{1} \wedge d x_{3}+a_{14} d x_{1} \wedge d x_{4}+a_{23} d x_{2} \wedge d x_{3}+a_{24} d x_{2} \wedge d x_{4}$, where $a_{13}, a_{14}, a_{23}, a_{24} \in \mathbb{Z}$ form a solution of the equation (4.2).

- If $\rho(A)=4$, then $\mathrm{H}^{1,1}(A, \mathbb{Z})$ is spanned by the forms

(i) $d x_{1} \wedge d x_{2}$;

(ii) $d x_{3} \wedge d x_{4}$;

(iii) $a_{13} d x_{1} \wedge d x_{3}+a_{14} d x_{1} \wedge d x_{4}+a_{23} d x_{2} \wedge d x_{3}+a_{24} d x_{2} \wedge d x_{4}$; and

(iv) $a_{13}^{\prime} d x_{1} \wedge d x_{3}+a_{14}^{\prime} d x_{1} \wedge d x_{4}+a_{23}^{\prime} d x_{2} \wedge d x_{3}+a_{24}^{\prime} d x_{2} \wedge d x_{4}$. 
Here, in (iii) and (iv) above, the collection integers

$$
a_{13}, a_{14}, a_{23}, a_{24}, a_{13}^{\prime}, a_{14}^{\prime}, a_{23}^{\prime}, a_{24}^{\prime} \in \mathbb{Z}
$$

form two solutions to the equation (4.2).

In particular, the Picard number $\rho(A)$ is described as

$$
\rho(A)= \begin{cases}2 & \text { if and only if } E_{1} \text { and } E_{2} \text { are not isogenous } \\ 3 & \text { if and only if } E_{1} \text { and } E_{2} \text { are isogenous and do not have CM } \\ 4 & \text { if and only if } E_{1} \text { and } E_{2} \text { are isogenous and have CM. }\end{cases}
$$

Turing to the case that

$$
A \simeq E_{1} \times \cdots \times E_{g}
$$

is the $g$-fold product of a collection of elliptic curves

$$
E_{i} \simeq \mathbb{C} / \Lambda_{i}
$$

$\tau_{i} \in \mathfrak{h}, i=1, \ldots, g$, the above discussion combined with the Theorem of the Cube, [20], allows for calculation of the Picard number $\rho(A)$. For example, the Picard number $\rho(A)$ is described as

$$
\rho(A)=g+\sum_{i=1}^{g-1} \sum_{j=i+1}^{g} \rho_{\text {new }}\left(\mathrm{E}_{i} \times \mathrm{E}_{j}\right)
$$

here

$$
\rho_{\text {new }}\left(\mathrm{E}_{i} \times \mathrm{E}_{j}\right)= \begin{cases}0 & \text { if and only if } \mathrm{E}_{i} \text { and } \mathrm{E}_{j} \text { are not isogenous } \\ 1 & \text { if and only if } \mathrm{E}_{i} \text { and } \mathrm{E}_{j} \text { are isogenous and do not have } \mathrm{CM} \\ 2 & \text { if and only if } \mathrm{E}_{i} \text { and } \mathrm{E}_{j} \text { are isogenous and have CM. }\end{cases}
$$

Finally, turning to the Brauer group of such a $g$-fold product of elliptic curves $A$, applying Corollary 4.5, it follows that $\operatorname{Br}(A)[r]$ is a free $\mathbb{Z} / r \mathbb{Z}$-module of rank $\left(\begin{array}{c}2 g \\ 2\end{array}\right)-\rho(A)$, where $\rho(A)$ is described in (4.3).

\section{Nondegenerate theta Groups And the Schrödinger Representations}

In this section, for later use, we discuss the weight 1 representation theory of nondegenerate theta groups. It is a special case of the general theory given in [15, Chapter 5], which generalizes previous work of Mumford [17, Section 1] and [18, Section 6]. For our purposes, we let $S:=\operatorname{Spec}(\mathbf{k})$ for $\mathbf{k}$ an algebraically closed base field.

Let $\pi: \mathrm{K} \rightarrow S$ be finite commutative group scheme, locally free of rank $r^{2}$, and fix a nondegenerate theta group over $\mathrm{K}$

$$
1 \rightarrow \mathbb{G}_{m} \rightarrow \mathbb{G} \rightarrow \mathrm{K} \rightarrow 1
$$


Equivalently, (5.1) is a central extension and the alternating form

$$
e: \mathrm{K} \times \mathrm{K} \rightarrow \mathbb{G}_{m},
$$

which is induced by the commutator of $\mathbb{G}$, is a perfect (nondegenerate) pairing.

Let $M$ be a $\mathbb{G}$-module. Restricting the $\mathbb{G}$-action to $\mathbb{G}_{m}$, yields the weight decomposition

$$
M=\bigoplus_{i \in \mathbb{Z}} M^{(i)}
$$

In (5.3),$M^{(i)}$ is the largest submodule of $M$ where $\mathbb{G}_{m}$ acts by the character $\lambda \mapsto \lambda^{i}$. The $\mathbb{G}$-module $M$ is said to be of weight $i \in \mathbb{Z}$ in case that $M=M^{(i)}$.

Fix a line bundle $\mathcal{L}$ on $\mathrm{K}$ so that

$$
\mathbb{G}=\operatorname{Isom}_{\mathcal{O}_{\mathrm{K}}-\bmod }\left(\mathcal{O}_{\mathrm{K}}, \mathcal{L}\right) .
$$

Put

$$
A^{(i)}(\mathbb{G})=\pi_{*}\left(\mathcal{L}^{\otimes-i}\right)
$$

and consider the natural action of $\mathbb{G}$ on its coordinate ring

$$
A(\mathbb{G})=\bigoplus_{i \in \mathbb{Z}} A^{(i)}(\mathbb{G})
$$

Note that each $A^{(i)}(\mathbb{G})$ is a locally free $\mathcal{O}_{S^{-}}$module of rank $r^{2}$.

The natural action of $\mathbb{G} \times \mathbb{G}$ on $A(\mathbb{G})$ respects the decomposition (5.4). Let $E^{(1)}$ be the $(\mathbb{G} \times \mathbb{G})$-module determined by $A^{(1)}(\mathbb{G})$ and $E_{+}^{(1)}$, respectively $E_{-}^{(1)}$, the $\mathbb{G}$-module obtained by restricting to $\{1\} \times \mathbb{G}$, respectively $\mathbb{G} \times\{1\}$, the action of $\mathbb{G} \times \mathbb{G}$ on $E^{(1)}$. Then $E_{+}^{(1)}$ has weight 1 whereas $E_{-}^{(1)}$ has weight -1 .

Lemma 5.1. In the above notation, $E^{(1)}$ is an irreducible $\mathbb{G} \times \mathbb{G}$-module. In particular, each $\mathbb{G} \times \mathbb{G}$-submodule of $E^{(1)}$ is of the form $I \cdot E^{(1)}$ where $I$ is an ideal of $\mathcal{O}_{S}$.

Proof. This is a special case of [15, Lemma 2.3, page 111].

Let $\operatorname{Rep}^{1}(\mathbb{G})$ be the Abelian category of weight one representations. Fix $V \in \operatorname{Rep}^{1}(\mathbb{G})$ and suppose that $V$ is locally free of rank $r$ as an $\mathcal{O}_{S^{-}}$-module. Let $V^{\vee}$ be the dual of $V$. In this context, there exists a natural homomorphism

$$
V \otimes_{\mathcal{O}_{S}} V^{\vee} \rightarrow A^{(1)}(\mathbb{G})=E^{(1)}
$$

which is defined by

$$
v \otimes v^{\vee} \in V \otimes V^{\vee} \mapsto\left(g \mapsto\left\langle g v, v^{\vee}\right\rangle\right) .
$$

This is a $\mathbb{G} \times \mathbb{G}$ morphism with $\mathbb{G} \times\{1\}$ acting by the given $\mathbb{G}$-representation and $\{1\} \times \mathbb{G}$ acting by the natural dual action.

The weight 1 representation theory of $\mathbb{G}$ is described as follows.

Theorem 5.2 ([15]). The following assertions hold true. 
(i) The natural homomorphism (5.5) is an isomorphism.

(ii) If $M$ is a $\mathbb{G}$-module with weight 1 , then the natural $\mathbb{G}$-morphism

$$
V \otimes \operatorname{Hom}_{\mathbb{G}}(V, M)^{\text {triv }} \rightarrow M
$$

defined by

$$
v \otimes u \mapsto u(v)
$$

is an isomorphism.

(iii) If $N$ is a quasi-coherent $\mathcal{O}_{S}$-module, then the natural $\mathbb{G}$-morphism

$$
N \mapsto \operatorname{Hom}_{\mathbb{G}}\left(V, V \otimes N^{\text {triv }}\right)
$$

defined by

$$
x \mapsto(v \mapsto v \otimes x)
$$

is an isomorphism.

Proof. This is a special case of [15, Theorem 2.4.2, page 112].

The regular weight 1, or Schrödinger Representations of $\mathbb{G}$ are determined by level and Lagrangian subgroups.

Definition 5.3. A level subgroup of $\mathbb{G}$ is a subgroup scheme $H \subseteq \mathbb{G}$, finite locally free over $S$ and such that $\mathrm{H} \cap \mathbb{G}_{m}=\{1\}$. A level subgroup is Lagrangian in case that it has rank $r$ over $S$.

The Schrödinger Representations are constructed as follows.

Proposition 5.4. Let $\mathrm{H} \subseteq \mathbb{G}$ be a level subgroup scheme of rank $r^{\prime}$ and put

$$
V=\left(E_{-}^{(1)}\right)^{\mathrm{H}}=\left(E^{(1)}\right)^{\{1\} \times \mathrm{H}} .
$$

Then $V$ is a $\mathbb{G}$-submodule of $E_{+}^{(1)}$, locally free of rank $r^{2} / r^{\prime}$ as a $\mathcal{O}_{S}$-module. In particular, if $\mathrm{H}$ is Lagrangian, then $V$ is locally free of rank $r$.

Proof. This is a special case of [15, Proposition 2.5.2, page 115].

Remark 5.5. When $r$ is not divisible by the characteristic of $\mathbf{k}$, then Lagrangian subgroups exist [17]. Further, in this context, the nature of higher weight representations of the nondegenerate theta groups is determined in [9].

\section{Proof of Theorem 1.1}

The purpose of this section is to prove Theorem 1.1. Our presentation follows Brion's suggestion [5, Remark 3.13]. For completeness, we restate Theorem 1.1 as Theorem 6.1 below. For the case of complex Abelian varieties, it refines [7, Theorem 1]. 
Theorem 6.1. Let $A$ be an Abelian variety over an algebraically closed field $\mathbf{k}$. Fix a positive integer $r$ which is not divisible by the characteristic of $\mathbf{k}$. Let $\operatorname{Br}(A)[r]$ be the $r$ torsion subgroup of the Brauer group of $A$. Then each class in $\operatorname{Br}(A)[r]$ may be represented by a homogeneous irreducible projective bundle.

Proof of Theorem 1.1 and [6.1. First, recall, as in [20, page 227], that if $M$ is a line bundle on $A$ and if

$$
\mathrm{K}(M):=\left\{x \in A: \tau_{x}^{*} M \simeq M\right\},
$$

then the commutator of the theta group

$$
\mathbb{G}(M):=\left\{(\phi, x): x \in \mathrm{K}(M) \text { and } \phi: \tau_{x}^{*} M \stackrel{\sim}{\rightarrow} M\right\},
$$

determines an alternating bilinear form

$$
e^{M}: \mathrm{K}(M) \times \mathrm{K}(M) \rightarrow \mathbb{G}_{m} .
$$

In what follows, if

$$
H \subseteq \mathrm{K}(M)
$$

is a subgroup scheme, then let $\left.e^{M}\right|_{H}$ denote the induced alternating form

$$
\left.e^{M}\right|_{H}: H \times H \rightarrow \mathbb{G}_{m}
$$

Now, as in Theorem 4.4 and Corollary 4.5, the Kummer sequence

$$
1 \rightarrow \mu_{r} \rightarrow \mathbb{G}_{m} \stackrel{\cdot r}{\rightarrow} \mathbb{G}_{m} \rightarrow 1
$$

induces an exact sequence

$$
0 \rightarrow \mathrm{NS}(A) / r \mathrm{NS}(A) \stackrel{\phi}{\rightarrow} \mathrm{H}_{\mathrm{et}}^{2}\left(A, \mu_{r}\right)=\operatorname{Hom}_{\mathbb{Z} / r \mathbb{Z}}\left(\bigwedge^{2} A[r], \mu_{r}\right) \stackrel{\psi}{\rightarrow} \mathrm{H}_{\mathrm{et}}^{2}\left(A, \mathbb{G}_{m}\right)[r] \rightarrow 0 .
$$

The map $\phi$ sends the class of a line bundle $L$ to the form $\left.e^{r L}\right|_{A[r]}$ and the map $\psi$ sends a form $e$ to the class of the Azumaya algebra

$$
\mathcal{A}:=\bigoplus_{\substack{\alpha \in A^{t}[r] \\ \sigma \in A[r]}} \alpha \cdot \mathbf{e}_{\sigma}
$$

In (6.1), we identify points $\alpha \in A^{t}[r]$ with the $r$-torsion line bundles $\alpha$ that they determine. Let

$$
e_{r}(\cdot, \cdot): A[r] \times A^{t}[r] \rightarrow \mu_{r}
$$

be the canonical pairing. Then the multiplication in (6.1) is defined, for local sections $s_{\alpha}$ of $\alpha$, and $s_{\beta}$ of $\beta$, by the condition that

$$
\left(s_{\alpha} \cdot \mathbf{e}_{\sigma}\right) \cdot\left(s_{\beta} \cdot \mathbf{e}_{\tau}\right)=\left(e_{r}(\beta, \sigma) \cdot a_{\sigma, \tau} \cdot s_{\alpha} \cdot s_{\beta}\right) \cdot \mathbf{e}_{\sigma+\tau} .
$$

Here

$$
\left\{a_{\sigma, \tau}\right\} \in Z^{2}\left(A[r], \mathbb{G}_{m}\right)
$$


is a 2-cocycle that has the property that

$$
e(\sigma, \tau)=a_{\sigma, \tau} \cdot a_{\tau, \sigma}^{-1} .
$$

The algebra (6.1) has rank $r^{2 g}$ and is free as a left module over the maximal étale subalgebra

$$
\mathcal{L}:=\bigoplus_{\alpha \in A^{t}[r]} \alpha \cdot \mathbf{e}_{0} .
$$

By [10, Corollary 5.5], it follows that

$$
[r]_{A}^{*} \mathcal{A} \simeq M_{r^{2 g}}\left(\mathcal{O}_{A}\right)
$$

Let $\mathrm{P}$ be the $\mathbb{P}^{r^{g}-1}$-bundle that corresponds to the Azumaya algebra (6.1). Then, following the terminology of [5, page 2493], $\mathrm{P}$ is trivialized by $[r]_{A}$. Indeed, this is implied by (6.2).

The above discussion implies that each class in $\operatorname{Br}(A)[r]$ is represented by a homogeneous $\mathbb{P}^{r^{g}-1}$-bundle which is trivialized by $[r]_{A}$. Write the fixed choice of such a bundle as

$$
\mathrm{P} \simeq A \times{ }^{A[r]} \mathbb{P}^{r^{g}-1}
$$

where the action of $A[r]$ on $\mathbb{P}^{r^{g}-1}$ is achieved via some projective representation

$$
\rho: A[r] \rightarrow \mathrm{PGL}_{r}
$$

as in [5, Proposition 3.9 (i)].

Let

$$
e_{\mathrm{P}, r}(\cdot, \cdot)=e(\cdot, \cdot): A[r] \times A[r] \rightarrow \mu_{r}
$$

be the alternating bilinear form that is determined by this trivialization, [5, pages 2494, 2495], and let

$$
A[r]^{\perp}=\{x \in A[r]: e(x, y)=1 \text { for all } y \in A[r]\}
$$

be the orthogonal complement of $A[r]$ with respect to $e$. Then

$$
\left[A[r]: A[r]^{\perp}\right]=d^{2}
$$

for $d$ the homogeneous index of the bundle $\mathrm{P}$ [5, page 2487]. It is the minimal rank of a homogeneous subbundle.

Let $S \subseteq \mathbb{P}^{r^{g}-1}$ be a linear subspace which is $A[r]$-stable and which is minimal for this property. Then

$$
\mathrm{P}_{1}:=A \times^{A[r]} \mathbb{P}^{d-1}
$$

is a homogeneous irreducible $\mathbb{P}^{d-1}$-bundle. In terms of the classification of Theorem 1.3 (i), it is obtained via the anti-affine extension

$$
1 \rightarrow A[r] / A[r]^{\perp} \rightarrow A / A[r]^{\perp} \rightarrow A / A[r]=A \rightarrow 1
$$

together with the induced faithful representation

$$
\rho_{1}: A[r] / A[r]^{\perp} \rightarrow \mathrm{PGL}_{r^{g}, d} \rightarrow \mathrm{PGL}_{d} .
$$


Here, $\mathrm{PGL}_{r, d} \subseteq \mathrm{PGL}_{r g_{-}}$is the maximal parabolic subgroup that stabilizes

$$
\mathbb{P}^{d-1} \simeq S \subseteq \mathbb{P}^{r^{g}-1} \text {. }
$$

Finally, it remains to show that $\mathrm{P}_{1}$ and $\mathrm{P}$ have the same class in $\operatorname{Br}(A)[r]$. To this end, $\mathrm{P}_{1} \cdot \mathrm{P}_{1}^{*}$ is the projectivization of a vector bundle. On the other hand, $\mathrm{P}_{1} \cdot \mathrm{P}_{1}^{*}$ is a subbundle of $\mathrm{P} \cdot \mathrm{P}_{1}^{*}$. It follows from [5, Lemma 3.12], that $\mathrm{P} \cdot \mathrm{P}_{1}^{*}$ is the projectivization of a vector bundle too.

\section{Homogeneous irreducible Severi-Brauer varieties over Abelian varieties}

Our main focus here is the study of homogeneous Severi-Brauer varieties over an Abelian variety $A$. Recall, that a $\mathbb{P}^{n-1}$-bundle, $p \nmid n$, over $A$ is homogeneous if it is isomorphic, as a bundle over $A$, to its pullback under all translations. A homogeneous $\mathbb{P}^{n-1}$-bundle is irreducible if it contains no non-trivial proper homogeneous subbundle [5, Proposition 3.5].

We describe the basic method of [5] for constructing homogeneous irreducible Severi-Brauer varieties over $A$. It is implicit in the proof of Corollary 2.4. In particular, each finite subgroup scheme $\mathrm{K} \subseteq A[n]$, with $\# \mathrm{~K}=n^{2}$ and $p \nmid n$, together with a faithful irreducible projective representation

$$
\rho: \mathrm{K} \rightarrow \mathrm{PGL}_{n}(\mathbf{k}),
$$

determines a homogeneous $\mathbb{P}^{n-1}$-bundle over $A$. Such bundles may be described as

$$
\mathrm{P}:=\left(A^{t} / \mathrm{K}^{t}\right)^{t} \times{ }^{\mathrm{K}} \mathbb{P}^{n-1} .
$$

In (7.2), $\mathrm{K}$ acts on $\mathbb{P}^{n-1}$ via the representation (7.1) and on $\left(A^{t} / \mathrm{K}^{t}\right)^{t}$ via translation. Here, we use the fact that

$$
\mathrm{K}^{t t}=\mathrm{K}
$$

is the kernel of the isogeny

$$
A=A^{t t} \leftarrow\left(A^{t} / \mathrm{K}^{t}\right)^{t}
$$

that is dual to

$$
A^{t} \rightarrow A^{t} / \mathrm{K}^{t}
$$

[14, Theorem 11.1].

As is a consequence of Proposition [5.4, such homogeneous $\mathbb{P}^{n-1}$-bundles are irreducible. For later use, we record the fact that the theory of the Schrödinger representations can be used to construct homogeneous irreducible $\mathbb{P}^{n-1}$-bundles over $A$.

Proposition 7.1. Let $A$ be an Abelian variety over an algebraically closed field $\mathbf{k}$. Let $\mathrm{K} \subseteq A[n]$ be a finite subgroup of rank $n^{2}$, for $n$ not divisible by the characteristic of $\mathbf{k}$, and consider a nondegenerate theta group over $\mathrm{K}$

$$
1 \rightarrow \mathbb{G}_{m} \rightarrow \mathbb{G} \rightarrow \mathrm{K} \rightarrow 1,
$$


with Schrödinger representation $(V, \rho)$ determined by a Lagrangian subgroup scheme $\mathrm{H} \subseteq \mathbb{G}$. Then $(V, \rho)$ determines a homogeneous irreducible $\mathbb{P}^{n-1}$-bundle over $A$. Conversely, each homogeneous irreducible $\mathbb{P}^{n-1}$-bundle over $A$ arises in this way.

Proof. Consider the faithful projective projective representation

$$
\rho: \mathrm{K} \rightarrow \mathrm{PGL}_{n}
$$

that is determined by $(V, \rho)$. Then, as in the above discussion, we obtain a $\mathbb{P}^{n-1}$-bundle over A

$$
\mathrm{P}_{(V, \rho)}:=\left(A^{t} / \mathrm{K}^{t}\right)^{t} \times{ }^{\mathrm{K}} \mathbb{P}^{n-1} .
$$

Here the quotient is taken with respect to the diagonal action of $\mathrm{K}$. In particular, $\mathrm{K}$ acts by

$$
k \cdot(x, v):=(k+x, \rho(k) \cdot v) .
$$

The fact that $\mathrm{P}_{(V, \rho)}$ is homogeneous is evident. That $\mathrm{P}_{(V, \rho)}$ is irreducible follows from Proposition 5.4. Finally, the converse follows via Corollary 2.4 and Theorem 6.1.

\section{Biextensions, Cubic structures And theta groups}

In this section, we briefly describe some concepts which pertain to biextensions, cubic structures and theta groups. More details can be found in [19], [3], and [15]. In Section 9, we use these concepts to study Severi-Brauer varieties and Azumaya algebras over Abelian varieties. For the basic structure theory of commutative groups schemes, over a fixed algebraically closed base field $\mathbf{k}$, we refer to [21].

Let $\mathrm{K}$ be a commutative group scheme over $\mathbf{k}$ and $\pi: L \rightarrow \mathrm{K}$ a $\mathbb{G}_{m}$-torsor. Over $\mathrm{K} \times \mathrm{K}$, we let

$$
\pi \hat{*} \pi: L \hat{*} L \rightarrow \mathrm{K} \times \mathrm{K}
$$

denote the $\mathbb{G}_{m}$-torsor defined by the condition that its fiber over

$$
(x, y) \in \mathrm{K} \times \mathrm{K}
$$

is described by

$$
\left.L \hat{*} L\right|_{x, y}=\left.\left.\left.L^{-1}\right|_{x} \otimes L^{-1}\right|_{y} \otimes L\right|_{x+y} .
$$

This torsor carries a canonical symmetry

$$
\xi_{x, y}:\left.\left.L \hat{*} L\right|_{x, y} \stackrel{\sim}{\rightarrow} L \hat{*} L\right|_{y, x} .
$$

Over $\mathrm{K} \times \mathrm{K} \times \mathrm{K}$, let $\Theta(L)$ denote the $\mathbb{G}_{m}$-torsor which has fiber over

$$
(x, y, z) \in \mathrm{K} \times \mathrm{K} \times \mathrm{K}
$$

given by

$$
\left.\Theta(L)\right|_{x, y, z}=\left.\left.\left.\left.\left.\left.\left.L\right|_{x} \otimes L\right|_{y} \otimes L\right|_{z} \otimes L^{-1}\right|_{x+y} \otimes L^{-1}\right|_{x+z} \otimes L^{-1}\right|_{y+z} \otimes L\right|_{x+y+z}
$$


There is a concept of a symmetric biextension structure for $L \hat{*} L$ as a $\mathbb{G}_{m}$-torsor over $\mathrm{K} \times \mathrm{K}$, [3, Section 1], [15, Chapter I.2.5], see also [19, Section 2]. We require two composition laws

$$
+_{1}: L \hat{*} L \times L \hat{*} L \rightarrow L \hat{*} L
$$

and

$$
+_{2}: L \hat{*} L \times L \hat{*} L \rightarrow L \hat{*} L .
$$

These laws are required to have the properties that

(i) for each $x \in \mathrm{K}$, via $+_{1}$, the pullback $\left.L \hat{*} L\right|_{x \times \mathrm{K}}$, of $L \hat{*} L$ over $x \times \mathrm{K}$ is a commutative group scheme and the induced morphism

$$
\left.L \hat{*} L\right|_{x \times \mathrm{K}} \rightarrow \mathrm{K}
$$

is a surjective homomorphism with kernel equal to $\mathbb{G}_{m}$;

(ii) for each $y \in \mathrm{K}$, via $+_{2}$, the pullback $\left.L \hat{*} L\right|_{\mathrm{K} \times y}$, of $L \hat{*} L$ over $\mathrm{K} \times y$ is a commutative group scheme and the induced morphism

$$
\left.L \hat{*} L\right|_{\mathrm{K} \times y} \rightarrow \mathrm{K}
$$

is a surjective homomorphism with kernel equal to $\mathbb{G}_{m}$; and

(iii) if $x, y, u, v \in L \hat{*} L$ have respective image in $\mathrm{K} \times \mathrm{K}$ given by

- $\pi \hat{*} \pi(x)=\left(b_{1}, c_{1}\right)$;

- $\pi \hat{*} \pi(y)=\left(b_{1}, c_{2}\right)$;

- $\pi \hat{*} \pi(u)=\left(b_{2}, c_{1}\right)$; and

- $\pi \hat{*} \pi(v)=\left(b_{2}, c_{2}\right)$,

then:

$$
\left(x+{ }_{1} y\right)+_{2}\left(u+{ }_{1} v\right)=\left(x+{ }_{2} u\right)+{ }_{1}\left(y+{ }_{2} v\right) .
$$

There is a concept of cubic structure for $L$ as a $\mathbb{G}_{m}$-torsor over K, [3, Section 2], [15, Chapter I.2.4]. This is a section $t$ of $\Theta(L)$, as a $\mathbb{G}_{m}$-torsor over $\mathrm{K} \times \mathrm{K} \times \mathrm{K}$, which is invariant under the natural action of the symmetric group on three letters and which is also a two cocycle in each pair of variables. We say that the pair $(L, t)$ is a cubic torsor. There is an evident concept of morphisms between cubic torsors. Let $\mathcal{C} u b\left(\mathrm{~K}, \mathbb{G}_{m}\right)$ denote the category of cubic torsors over K.

The following remark clarifies the relation amongst cubic and symmetric biextension structures.

Proposition 8.1 ([15, Proposition 2.5.4]). Let K be a commutative group scheme over an algebraically closed based field $\mathbf{k}$. Let $\pi: L \rightarrow \mathrm{K}$ be a $\mathbb{G}_{m}$-torsor. To give a symmetric biextension structure on the $\mathbb{G}_{m}$-torsor $L \hat{*} L$, is equivalent to giving a cubic structure on $L$ as a $\mathbb{G}_{m}$-torsor over $\mathrm{K}$.

Proof. This is a special case of [15, Proposition 2.5.4], see also [3, Section 2]. Nevertheless, we include a proof here. Specifically, we explain how cubic structures on a $\mathbb{G}_{m}$-torsor $L$ are 
related to symmetric biextension structures on $L \hat{*} L$. Important to that discussion is the following canonical commutative diagram

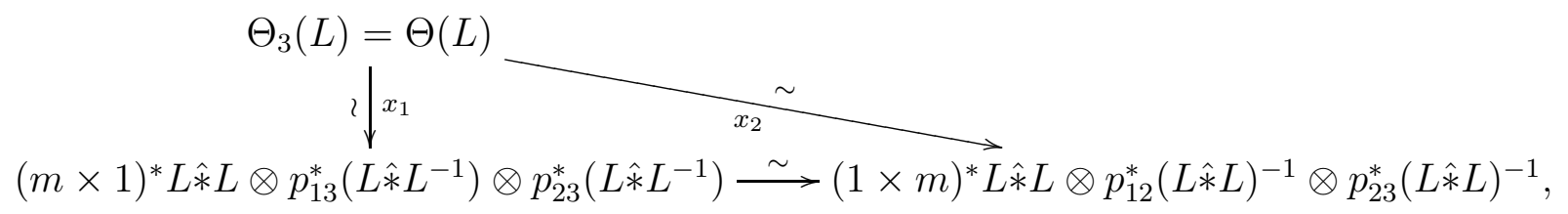

[3. Equation 2.1.10]. In the above diagram, $m$ denotes multiplication in the group law, $p_{i j}$ denotes projection onto the $i, j$-factor and $x_{i}$, for $i=1,2$, denotes the evident natural isomorphism. These morphisms $x_{i}$ are induced, respectively, by the natural trivialization morphisms $\left.\left.\mathcal{O}_{\mathrm{K}} \stackrel{\sim}{\rightarrow} L^{-1}\right|_{x_{1}} \otimes L\right|_{x_{1}}$ and $\left.\left.\mathcal{O}_{\mathrm{K}} \stackrel{\sim}{\rightarrow} L^{-1}\right|_{x_{2}} \otimes L\right|_{x_{2}}$.

In particular, to begin with, suppose given a cubic structure $t$ on $\Theta(L)$. We then deduce, using the above canonical commutative diagram, that $t$ determines a biextension structure on $L \hat{*} L$. This biextension structure is compatible with the canonical symmetry

$$
\xi_{x, y}:\left.\left.L \hat{*} L\right|_{x, y} \stackrel{\sim}{\rightarrow} L \hat{*} L\right|_{y, x} .
$$

Conversely, suppose given a symmetric biextension structure on the $\mathbb{G}_{m}$-torsor $L \hat{*} L$. Especially, we are given sections $s_{1}$ and $s_{2}$ of, the respective, $\mathbb{G}_{m}$-torsors over $\mathrm{K}^{3}$

$$
(m \times 1)^{*} L \hat{*} L \otimes p_{13}^{*} L \hat{*} L^{-1} \otimes p_{23}^{*} L \hat{*} L^{-1}
$$

and

$$
(1 \times m)^{*} L \hat{*} L \otimes p_{12}^{*} L \hat{*} L^{-1} \otimes p_{13}^{*} L \hat{*} L^{-1}
$$

these sections are compatible with the canonical commutative diagram

$$
s_{1}=s_{2} .
$$

Further, since these sections correspond to the partial group law structure on $L \hat{*} L$, considered as a biextension, they are commutative and associative. Put

$$
t=x_{1}^{-1}\left(s_{1}\right)=x_{2}^{-1}\left(s_{2}\right) .
$$

The point now is to check that this section of $\Theta(L)$ determines a cubic structure.

For example, over $\mathrm{K}^{4}$, we consider the $\mathbb{G}_{m}$-torsor

$$
p_{234}^{*} \Theta(L) \otimes m_{12}^{*} \Theta(L)^{-1} \otimes m_{23}^{*} \Theta(L) \otimes p_{124} \Theta(L)^{-1},
$$

which has fiber

$$
\left.\left.\left.\left.\Theta(L)\right|_{y, z, w} \otimes \Theta(L)^{-1}\right|_{x+y, z, w} \otimes \Theta(L)\right|_{x, y+z, w} \otimes \Theta(L)^{-1}\right|_{x, y, w}
$$

over

$$
(x, y, z, w) \in \mathrm{K}^{4} .
$$

(Here, we also use subscripts to denote the evident maps determined by the various factors of $\mathrm{K}^{4}$.) This above torsor has a canonical section which also induces a canonical isomorphism

$$
\left.\left.\left.\left.\Theta(L)\right|_{x+y, z, w} \otimes \Theta(L)\right|_{x, y, w} \stackrel{\sim}{\rightarrow} \Theta(L)\right|_{y, z, w} \otimes \Theta(L)\right|_{x, y+z, w} .
$$


As in [3, Section 2.5], the condition that $s_{1}$ is associative, for example, is equivalent to the assertion that the section

$$
t(y, z, w) \cdot t(x+y, z, w)^{-1} \cdot t(x, y+z, w) \cdot t(x, y, w)^{-1}
$$

(over $\mathrm{K}^{4}$ ) coincides with the canonical section, that is

$$
t(x+y, z, w) \cdot t(x, y, w)=t(y, z, w) \cdot t(x, y+z, w) .
$$

Similarly, the associativity of $s_{2}$ is expressed in terms of the canonical isomorphism

$$
\left.\left.\left.\left.\Theta(L)\right|_{x, y+z, w} \otimes \Theta(L)\right|_{x, y, z} \stackrel{\sim}{\rightarrow} \Theta(L)\right|_{x, z, w} \otimes \Theta(L)\right|_{x, y, z+w}
$$

which pertains to

$$
t(x, y+z, w) \cdot t(x, y, z)=t(x, z, w) \cdot t(x, y, z+w) .
$$

Finally, as in [3, page 18], we have an additional canonical commutative diagram

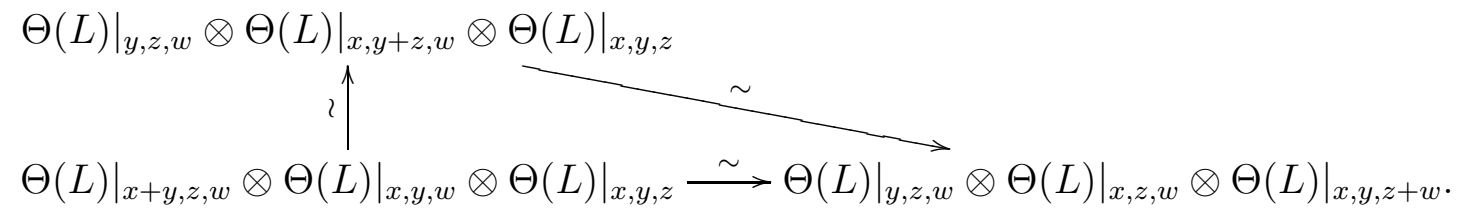

This diagram implies that

$$
t(x+y, z, w) \cdot t(x, y, w) \cdot t(x, y, z)=t(y, z, w) \cdot t(x, z, w) \cdot t(x, y, z+w) .
$$

(Here we also use compatibility of the partial group laws $s_{1}$ and $s_{2}$.)

It remains to check invariance under the natural action of $\mathfrak{S}_{3}$, the symmetric group on three letters. To this end, for each $\sigma \in \mathfrak{S}_{3}$, we have the canonical isomorphism

$$
x_{\sigma}: \sigma^{*} \Theta(L) \stackrel{\sim}{\rightarrow} \Theta(L)
$$

which is induced by the group law on K. Put

$$
t=t\left(x_{1}, x_{2}, x_{3}\right)
$$

and let

$$
t\left(x_{\sigma(1)}, x_{\sigma(2)}, x_{\sigma(3)}\right)=\sigma^{*} t
$$

be the section of $\sigma^{*} \Theta(L)$ which is induced by $t$. The fact that $s_{1}$ and $s_{2}$ are commutative, then imply the relations

$$
x_{(12)}\left(t\left(x_{2}, x_{1}, x_{3}\right)\right)=t\left(x_{1}, x_{2}, x_{3}\right)
$$

and

$$
x_{(23)}\left(t\left(x_{1}, x_{3}, x_{2}\right)\right)=t\left(x_{1}, x_{2}, x_{3}\right) .
$$

More generally, it holds true that

$$
x_{\sigma}\left(t\left(x_{\sigma(1)}, x_{\sigma(2)}, x_{\sigma(3)}\right)\right)=t\left(x_{1}, x_{2}, x_{3}\right),
$$

for each permutation $\sigma \in \mathfrak{S}_{3}$. In particular, the pair $(L, t)$ is an object of $\mathcal{C} u b\left(\mathrm{~K}, \mathbb{G}_{m}\right)$. 
Next, we consider central extensions. By a theta group over K, we mean a central extension

$$
1 \rightarrow \mathbb{G}_{m} \rightarrow \mathbb{G} \rightarrow \mathrm{K} \rightarrow 0
$$

of group schemes. Each such extension determines a well-defined alternating form

$$
e: \mathrm{K} \times \mathrm{K} \rightarrow \mathbb{G}_{m}
$$

which is determined by

$$
e\left(a^{\prime}, b^{\prime}\right)=[a, b]=a b a^{-1} b^{-1},
$$

for $a, b \in \mathbb{G}$ lying over $a^{\prime}, b^{\prime} \in \mathrm{K}$.

Here, $[\cdot, \cdot]$ is the commutator of the extension (8.1). In case that the form (8.2) is a perfect pairing, then we say that the extension (8.1) is nondegenerate. In this case, the group scheme $\mathrm{K}$ is finite and the pairing (8.2) induces an isomorphism amongst $\mathrm{K}$ and its Cartier dual. The collection of such central extensions (8.1), for $\mathrm{K}$ a given commutative group scheme, form a category which we denote by $\mathcal{C} \operatorname{ent}\left(\mathrm{K}, \mathbb{G}_{m}\right)$. The categories $\mathcal{C} e n t\left(\mathrm{~K}, \mathbb{G}_{m}\right)$ and $\mathcal{C} u b\left(\mathrm{~K}, \mathbb{G}_{m}\right)$ are related as follows.

Proposition 8.2 ([15, page 24]). Let $\mathrm{K}$ be a commutative group scheme. The category $\mathcal{C}$ ent $\left(\mathrm{K}, \mathbb{G}_{m}\right)$ is equivalent to the category of couples $((L, t), \sigma)$ where $(L, t)$ is an object of $\mathcal{C} u b\left(\mathrm{~K}, \mathbb{G}_{m}\right)$ and where $\sigma$ is a trivialization of the corresponding symmetric biextension structure for $L \hat{*} L$, as a $\mathbb{G}_{m}$-torsor over $\mathrm{K} \times \mathrm{K}$.

Proof. This is a special case of [15, page 22-24], compare also with [3, Section 2]. Here we indicate the main points of the argument. With this in mind, suppose that $(L, t)$ is a cubic $\mathbb{G}_{m}$-torsor over K. Let $\sigma$ be a trivialization of $\Theta_{2}(L)$. For each $x \in \mathrm{K}$ and each $y \in \mathrm{K}$, we have a section $\sigma(x, y)$ of

$$
\left.\left.\left.L\right|_{x+y} \otimes L^{-1}\right|_{x} \otimes L^{-1}\right|_{y}
$$

together with an isomorphism

$$
\left.\left.\left.L\right|_{x} \otimes L\right|_{y} \stackrel{\sim}{\rightarrow} L\right|_{x+y}
$$

This isomorphism (8.3) then defines a product law

$$
*: L \times L \rightarrow L
$$

that is compatible with addition and the given $\mathbb{G}_{m}$-action on $L$. The product law (8.4) can be described explicitly in terms of the trivialization $\sigma(\cdot, \cdot)$.

For instance, if $x, y \in \mathrm{K},\left.u \in L\right|_{x}$ and $\left.v \in L\right|_{y}$, then

$$
\left.u * v \in L\right|_{x+y}
$$

is defined by the condition that

$$
\sigma(x, y)=\left.(u * v) \otimes u^{-1} \otimes v^{-1} \in \Theta_{2}(L)\right|_{x+y} .
$$

By assumption, the section $\sigma$ is compatible with the biextension structure $t$ of $\Theta_{2}(L)$. 
From this viewpoint, that $\sigma$ is compatible with the first composition law means that

$$
\left.\left.\sigma(x, z) \otimes \sigma(y, z) \in \Theta_{2}(L)\right|_{x, z} \otimes \Theta_{2}(L)\right|_{y, z}
$$

is sent to

$$
\left.\sigma(x+y, z) \in \Theta_{2}(L)\right|_{x+y, z}
$$

via the condition that

$$
(u * w) \otimes u^{-1} \otimes w^{-1} \otimes(v * w) \otimes v^{-1} \otimes w^{-1} \otimes t(x, y, z)=((u * v) * w) \otimes(u * v)^{-1} \otimes w^{-1} .
$$

Here, we have that $\left.u \in L\right|_{x},\left.v \in L\right|_{y},\left.w \in L\right|_{z}$ with $x \in \mathrm{K}, y \in \mathrm{K}$ and $z \in \mathrm{K}$.

We then note

$$
t(x, y, z)=((u * v) * w) \otimes(u * v)^{-1} \otimes(u * w)^{-1} \otimes(v * w)^{-1} \otimes u \otimes v \otimes w .
$$

Similarly, compatibility with the second group law means

$$
t(x, y, z)=(u *(v * w)) \otimes(u * v)^{-1} \otimes(u * w)^{-1} \otimes(v * w)^{-1} \otimes u \otimes v \otimes w .
$$

Finally, by comparison

$$
(u * v) * w=u *(v * w) .
$$

In particular, (8.7) implies that the product law $*$ on $L \times L$ so defined is associative. Further, the section of $L$ at the origin induced by the cubic structure $t$ is trivial; the composition

$$
\left.\left.\left.\left.\mathbb{G}_{m} \otimes L\right|_{y} \stackrel{\sim}{\rightarrow} L\right|_{0} \otimes L\right|_{y} \stackrel{*}{\rightarrow} L\right|_{y}
$$

is the natural action of $\mathbb{G}_{m}$ on $\left.L\right|_{y}$.

In brief, we have shown that the cubic torsor $(L, t)$ over $\mathrm{K}$ is given, via the trivialization $\sigma$, the structure of a group. More precisely, $L$ is an object of $\mathcal{C}$ ent $\left(\mathrm{K}, \mathbb{G}_{m}\right)$. Finally, the product law $*$ can be viewed as a left action of $L$ on itself. The formulas (8.5) and (8.6) allow for the cubic structure $t$ to be reconstructed from this group law together with the fixed trivialization $\sigma$. The equivalence desired by the proposition now follows.

Proposition 8.2 allows for an alternative formulation of Theorem 1.3 and Corollary 2.3 .

Theorem 8.3. Let $A$ be an Abelian variety over an algebraically closed field $\mathbf{k}$. Fix an integer $n$ that is not divisible by the characteristic of $\mathbf{k}$. Then the homogeneous rank $n$ Severi-Brauer varieties over A are classified by the choice of an anti-affine extension

$$
1 \rightarrow H \rightarrow G \rightarrow A \rightarrow 1
$$

together with a pair $((L, t), \sigma)$ where $(L, t)$ is an object of $\mathcal{C} u b\left(H, \mathbb{G}_{m}\right)$ and where $\sigma$ is a trivialization of the corresponding symmetric biextension structure for $L \hat{*} L$ as a $\mathbb{G}_{m}$-torsor over $H \times H$. 
Proof. By Theorem [1.3, the homogeneous $\mathbb{P}^{n-1}$-bundles over $A$ are classified by the choice of such an anti-affine extension (8.8) together with a faithful projective representation

$$
\rho: H \rightarrow \mathrm{PGL}_{n} .
$$

But such a faithful projective representation (8.9) is equivalent to the choice of a central extension of the form (8.1) with $\mathrm{K}$ replaced by $H$. Thus the conclusion desired by Theorem 8.3 follows from that of Proposition 8.2 .

Motivated by Proposition 8.2 and Theorem 8.3, we formulate the concept of nondegenerate cubic torsor (see Definition 8.4 below). The main point is to identify those structures on cubic torsors which correspond to nondegenerate theta groups. We use this formalism in the statement of Theorem 1.2 .

Definition 8.4. Let $\mathrm{K}$ be a finite commutative group scheme with rank $\# \mathrm{~K}=n^{2}$ not divisible by the characteristic of $\mathbf{k}$. We say that a couple $((L, t), \sigma)$, where $(L, t)$ is an object of $\mathcal{C} u b\left(\mathrm{~K}, \mathbb{G}_{m}\right)$ and where $\sigma$ is a trivialization of the corresponding symmetric biextension $L \hat{*} L$, as a $\mathbb{G}_{m}$-torsor over $\mathrm{K} \times \mathrm{K}$, is nondegenerate if the central extension to which it corresponds, via Proposition 8.2, is nondegenerate. By abuse of terminology we also simply say that $((L, t), \sigma)$ is a nondegenerate cubic torsor.

\section{Characterizing the homogeneous irreducible Severi-Brauer varieties in TERMS OF CUBIC STRUCTURES}

Our main goal here is to prove

Theorem 9.1. Let $A$ be an Abelian variety over an algebraically closed field $\mathbf{k}$. Fix a positive integer $n$, with $p:=\operatorname{char}(\mathbf{k}) \nmid n$. Then the homogeneous irreducible $\mathbb{P}^{n-1}$-bundles over $A$ are classified by pairs

$$
(\mathrm{K},((L, t), \sigma)),
$$

where $\mathrm{K} \subseteq A[n]$ is a subgroup of rank $n^{2}$ and where $((L, t), \sigma)$ is a nondegenerate cubic torsor. In particular, $(L, t)$ is an object of $\mathcal{C} u b\left(\mathrm{~K}, \mathbb{G}_{m}\right), \sigma$ is a trivialization of the biextension structure on $\Lambda(L)$ which is induced by $t$ and the central extension corresponding to $((L, t), \sigma)$ is nondegenerate.

In our proof of Theorem 9.1, we require the elementary divisor (or invariant factor) theorem for nondegenerate alternating forms

$$
e: \mathrm{K} \times \mathrm{K} \rightarrow \mathbb{G}_{m} .
$$

Here, $\mathrm{K} \subseteq A[n]$ is a subgroup scheme of rank $n^{2}$ and $p \nmid n$. We recall the main points following [17, page 294] closely (see also [5, Remark 3.2]).

- Each nondegenerate alternating form

$$
e: \mathrm{K} \times \mathrm{K} \rightarrow \mathbb{G}_{m}
$$


determines a sequence of positive integers

$$
\delta=\left(d_{1}, d_{2}, \ldots, d_{k}\right),
$$

with

$$
d_{i+1} \mid d_{i}, \text { and } d_{i}>1
$$

so that

$$
\mathrm{K}=\bigoplus_{i=1}^{k}\left(\mathbb{Z} / d_{i} \mathbb{Z}\right)^{\oplus 2}
$$

- We can write

$$
\mathrm{K}=\mathrm{K}(\delta) \oplus \mathrm{K}(\delta)^{t}
$$

with

$$
\mathrm{K}(\delta)=\bigoplus_{i=1}^{k} \mathbb{Z} / d_{i} \mathbb{Z}
$$

and

$$
\mathrm{K}(\delta)^{t}=\operatorname{Hom}\left(\mathrm{K}(\delta), \mathbb{G}_{m}\right),
$$

the Cartier dual of $\mathrm{K}(\delta)$. The identification

$$
\mathrm{K}(\delta)=\mathrm{K}(\delta)^{t}
$$

is induced by the alternating form $e$.

- Put

$$
\mathbb{G}(\delta)=\mathbb{G}_{m} \times \mathrm{K}(\delta) \times \mathrm{K}(\delta)^{t}
$$

with group law

$$
(\alpha, x, \ell) \cdot\left(\alpha^{\prime}, x^{\prime}, \ell^{\prime}\right)=\left(\alpha \cdot \alpha^{\prime} \cdot \ell^{\prime}(x), x+x^{\prime}, \ell+\ell^{\prime}\right) .
$$

Then $\mathbb{G}(\delta)$ is a nondegenerate central extension

$$
1 \rightarrow \mathbb{G}_{m} \rightarrow \mathbb{G}(\delta) \rightarrow \mathrm{K} \rightarrow 0 ;
$$

it is the nondegenerate central extension determined by the nondegenerate alternating form (9.1). Conversely, the commutator of each nondegenerate central extension

$$
1 \rightarrow \mathbb{G}_{m} \rightarrow \mathbb{G} \rightarrow \mathrm{K} \rightarrow 0
$$

determines a nondegenerate alternating form, with shape (9.1).

- By considering the vector $\delta$, it follows that the nondegenerate central extension (9.3) is equivalent to the nondegenerate central extension (9.2). Finally, our discussion implies, that each nondegenerate alternating form (9.1) determines an irreducible weight 1 representation (namely that of $\mathbb{G}(\delta)$ for $\delta$ the elementary divisor vector of $e$ ). Conversely, for a given such vector $\delta$, there is a unique alternating form and irreducible weight 1 representation of this shape.

We now prove Theorem 9.1. 
Proof of Theorem 9.1. By Proposition 7.1, the homogeneous irreducible $\mathbb{P}^{n-1}$-bundles over $A$ have the form

$$
\mathrm{P}=\left(A^{t} / \mathrm{K}^{t}\right)^{t} \times{ }^{\mathrm{K}} \mathrm{P}^{n-1},
$$

for $\mathrm{K} \subseteq A[n]$ a subgroup with $\# \mathrm{~K}=n^{2}$ and where $\mathrm{K}$ acts on $\mathbb{P}^{n-1}$ by an irreducible faithful weight one representation. In particular, such a $\mathbb{P}^{n-1}$-bundle $\mathrm{P}$ is determined by the weight 1 representation of a nondegenerate theta group

$$
1 \rightarrow \mathbb{G}_{m} \rightarrow \mathbb{G} \rightarrow \mathrm{K} \rightarrow 1 \text {. }
$$

Thus, in light of Definition 8.4, Theorem 9.1 then follows upon applying Proposition 8.1 and Proposition 8.2 .

Finally, we use Theorem 1.1 and Theorem 9.1 to prove Theorem 1.2 .

Proof of Theorem 1.2. By Theorem 1.1, each class in $\operatorname{Br}(A)[r]$ is represented by a homogeneous irreducible Severi-Brauer variety. In turn, by Theorem 9.1, this means that each class in $\operatorname{Br}(A)[r]$ is represented by a nondegenerate pair $((L, t), \sigma)$. Equivalently, using the language of Definition [8.4, each class in $\operatorname{Br}(A)[r]$ is represented by a nondegenerate cubic torsor.

\section{REFERENCES}

[1] M. F. Atiyah, Vector bundles over an elliptic curve, Proc. London Math. Soc. (3) 7 (1957), 414-452.

[2] V. G. Berkovich, The Brauer group of abelian varieties, Funkcional Anal. i Priložen. 6 (1972), no. 3, $10-15$.

[3] L. Breen, Fonctions thêta et théorème du cube, Springer-Verlag, Berlin, 1983.

[4] M. Brion, Anti-affine algebraic groups, J. Algebra 321 (2009), no. 3, 934-952.

[5] _ Homogeneous projective bundles over abelian varieties, Algebra and Number Theory 7 (2013), no. $10,2475-2510$.

[6] Homogeneous vector bundles over varieties via representation theory, Represent. Theory $\mathbf{2 4}$ (2020), 85-114.

[7] G. Elencwajg and M. S. Narasimhan, Projective bundles on a complex torus, J. Reine Angew. Math. 340 (1983), 1-5.

[8] P. Gille and T. Szamuely, Central simple algebras and Galois cohomology, Cambridge University Press, Cambridge, 2006.

[9] N. Grieve, Refinements to Mumford's theta and adelic theta groups, Ann. Math. Qué. 38 (2014), no. 2, 145-167.

[10] A. Grothendieck, Le groupe de Brauer. I. Algèbres d'Azumaya et interprétations diverses, Séminaire Bourbaki, Vol. 9, Soc. Math. France, Paris, 1995.

[11] R. T. Hoobler, Brauer groups of abelian schemes, Ann. Sci. École Norm. Sup. (4) 5 (1972), 45-70.

[12] H. Kaji, On the vector bundles whose endomorphisms yield Azumaya algebras of cyclic type, J. Algebra 117 (1988), no. 2, 297-326.

[13] J. S. Milne, Étale cohomology, Princeton University Press, Princeton, N.J., 1980.

[14] _ Abelian varieties, Arithmetic geometry (Storrs, Conn., 1984), Springer, New York, 1986, pp. $103-150$.

[15] L. Moret-Bailly, Pinceaux de variétés abéliennes, Astérisque (1985), no. 129, 266. 
[16] S. Mukai, Semi-homogeneous vector bundles on an Abelian variety, J. Math. Kyoto Univ. 18 (1978), no. 2, 239-272.

[17] D. Mumford, On the equations defining abelian varieties. I, Invent. Math. 1 (1966), 287-354.

[18] _ On the equations defining abelian varieties. II, Invent. Math. 3 (1967), 75-135.

[19] _ Bi-extensions of formal groups, Algebraic Geometry (Internat. Colloq., Tata Inst. Fund. Res., Bombay, 1968), Oxford Univ. Press, London, 1969, pp. 307-322.

[20] _ Abelian varieties, Published for the Tata Institute of Fundamental Research, Bombay; Oxford University Press, London, 1970.

[21] F. Oort, Commutative group schemes, LNM, no. 15, Springer-Verlag, 1966.

[22] B. Poonen and E. Rains, Self cup products and the theta characteristic torsor, Math. Res. Lett. 18 (2011), no. 6, 1305-1318.

[23] A. N. Skorobogatov and Y. G. Zarhin, A finiteness theorem for the Brauer group of abelian varieties and K3 surfaces, J. Algebraic Geom. 17 (2008), no. 3, 481-502.

[24] 666 (2012), 115-140.

[25] _ Kummer varieties and their Brauer groups, Pure Appl. Math. Q. 13 (2017), no. 2, 337-368.

School of Mathematics and Statistics, 4302 Herzberg Laboratories, Carleton University, 1125 Colonel By Drive, Ottawa, ON, K1S 5B6, Canada

Département de mathématiques, Université du Québec á Montréal, Local PK-5151, 201 Avenue du Président-Kennedy, Montréal, QC, H2X 3Y7, Canada

Email address: nathan.m.grieve@gmail.com 